\title{
Axillary and internal mammary sentinel lymph node biopsy in male breast cancer patients: case series and review
}

This article was published in the following Dove Press journal:

OncoTargets and Therapy

17 June 2015

Number of times this article has been viewed

\author{
Xiaoshan $\mathrm{Cao}^{1,2}$ \\ Chunjian Wang' \\ Yanbing Liu' \\ Pengfei Qiu' \\ Binbin Cong ${ }^{1,2}$ \\ Yongsheng Wang' \\ 'Breast Cancer Center, Shandong \\ Cancer Hospital and Institute, Jinan, \\ Shandong, People's Republic of China; \\ ${ }^{2} \mathrm{~S} c h o o l$ of Medicine and Life Sciences. \\ Jinan University-Shandong Academy \\ of Medical Sciences, Jinan, Shandong, \\ People's Republic of China
}

\begin{abstract}
Male breast cancer (MBC) is considered as a rare disease that accounts for less than $1 \%$ of all breast cancers, and its treatment has been based on the evidence available from female breast cancer. Axillary sentinel lymph node biopsy (SLNB) is now regarded as the standard of care for both female and male patients without clinical and imaging evidence of axillary lymph node metastases, while internal mammary SLNB has rarely been performed. Internal mammary chain metastasis is an independent prognostic predictor. Internal mammary SLNB should be performed to complete nodal staging and guide adjuvant therapy in MBC patients with preoperative lymphoscintigraphic internal mammary chain drainage. We report both axillary and internal mammary SLNB in two cases with MBC. Internal mammary sentinel lymph node did contain metastasis in one case.
\end{abstract}

Keywords: male breast cancer, internal mammary lymph node, sentinel lymph node biopsy, case report

\section{Introduction}

Male breast cancer (MBC) is an uncommon phenomenon, accounting for less than 1\% of all breast cancer with an annual incidence of $1 / 10^{5}$ which was reported on during the 2014 San Antonio Breast Cancer Symposium. ${ }^{1}$ Because of its rarity, MBC has not been studied as extensively as female breast cancer (FBC), and its treatment has largely been based on that of FBC.

In clinically node-negative $\mathrm{FBC}$, several large randomized prospective trials have shown that axillary sentinel lymph node biopsy (ASLNB) substantially reduces the surgical morbidity compared to that of axillary lymph node dissection (ALND). ${ }^{2,3}$ The feasibility and accuracy of ASLNB replacing ALND have also been proved in MBC. ${ }^{4}$ Internal mammary sentinel lymph node biopsy (IM-SLNB) has not been performed routinely and has remained a subject of discussion both in FBC and MBC, and it has been reported even less in MBC. Four MBC patients were treated in our center from April 2014 to February 2015, and all of them received ASLNB, while internal mammary sentinel lymph node (IMSLN) was detected and investigational IM-SLNB performed successfully in two MBC patients. Here we report these two cases receiving ASLNB and IM-SLNB.

\section{Case report I}

Breast Cancer Center, Shandong Cancer Hospital and Institute, No 440, Jiyan Road, Jinan, Shandong, 250II7, People's Republic of China

Email wangysh2008@aliyun.com
Our first case was a 65 -year-old man with a painless lump in his left breast for more than 5 years. He was admitted to our hospital in October 2014. Open-biopsy had been performed 1 week prior in another hospital, and invasive ductal carcinoma of $2.4 \mathrm{~cm}$ and grade II-III 
was confirmed by routine pathology. Pathology consultation in our hospital indicated: (left) invasive ductal carcinoma, grade III, positive by immunohistochemistry (IHC) for estrogen receptor (ER) ( $>90 \%$ of nuclei stained) and progesterone receptor (PR) (about 10\% of nuclei stained) and negative for HER-2 by IHC. The average Ki-67 labeling index was 40\%. An elastic, well-defined and mobile axillary lymph node (ALN) about 1.0 $\mathrm{cm}$ in diameter was palpable in his left axilla. Ultrasonography revealed several enlarged ALNs with relatively normal structure, and ultrasound-guided fine needle aspiration was performed on the largest one $(1.2 \times 0.6 \mathrm{~cm})$ with benign cytological finding.

Thirty-seven $\mathrm{MBq}$ of ${ }^{99 \mathrm{~m}}$ Tc-labeled sulfur colloid ( ${ }^{99 \mathrm{~m}} \mathrm{Tc}-\mathrm{SC}$ ) in $1.2 \mathrm{~mL}$ volume intraparenchymal injections at the 6 and 12 o'clock positions about $1.0 \mathrm{~cm}$ from areola under ultrasonographic guidance 17 hours before surgery, and preoperative lymphoscintigraphy revealed no radioactive IMSLN. Methylene blue ( $2 \mathrm{~mL}$ ) was injected subcutaneously away from the open-biopsy cavity 10 minutes before surgery. The first axillary sentinel lymph node (ASLN) was found with blue dye combining ${ }^{99 \mathrm{~m} T c-S C}$, and the other three ASLNs were found with ${ }^{99 m}$ Tc-SC only. All ASLNs were evaluated with intraoperative frozen section histology and touch imprint cytology, and only the first one was found to be positive for metastasis. So a complete ALND was performed. After total mastectomy and ALND, the internal mammary chain area was explored with the hand-held gamma probe in search of radioactivity and a "hot spot" was assessed in the third intercostal space. The IM-SLNB was performed using the mastectomy incision. From the sternal border in a lateral direction $3.0 \mathrm{~cm}$ to $4.0 \mathrm{~cm}$, the pectoral major muscle fibers were separated to expose the posterior intercostal space. The external and internal intercostal muscles were divided transversally from the sternal border. In this procedure, particular care must be taken to avoid injury to the inferior parietal pleura and the internal mammary vessels. The IMSLN, just on the outside of the internal mammary artery, was removed easily and the procedure lasted only 10 minutes. The IMSLN was about $5 \mathrm{~mm}$ in diameter and the ex vivo counts were 35 . Then, the area was explored in search of radioactivity that was constantly assessed with the gamma-detecting probe to make sure that there were no residual areas of high radioactivity. Finally IMSLN was evaluated histologically with routine pathology. The IMSLN did not contain metastasis after definitive pathology. The final pathological stage for this male patient was pT2N1aM0 IIB.

\section{Case report 2}

Our second case was a 56-year-old man who was admitted to our hospital in February 2015 with a painless lump about $2 \times 1.5 \mathrm{~cm}$ in the left breast two years and a half. Preoperative core needle biopsy confirmed invasive ductal carcinoma, grade II. It was positive by IHC for ER (50\% of nuclei stained) and PR (about 20\% of nuclei stained) and negative for HER-2 by IHC. The average Ki-67 labeling index was $30 \%$. No lymph node was palpable in his left axilla and the ultrasonography also revealed no enlargement of ALNs.

Thirty-seven MBq of ${ }^{99 m}$ Tc-SC was injected as in the first case and preoperative lymphoscintigraphy revealed that there was a "hot spot" in his second intercostal space. Blue dye was injected subcutaneously around the tumor. Two ASLNs were found with blue dye combined with ${ }^{99 \mathrm{~m}} \mathrm{Tc}-\mathrm{SC}$. Intraoperative frozen section histology and touch imprint cytology showed that both of them were positive. Then a complete ALND was performed. After total mastectomy and ALND, the IMSLN was found by the hand-held gamma probe in the second intercostal space as the lymphoscintigraphy revealed, and it was removed through the intercostal space. The IMSLN was about $6 \mathrm{~mm}$ in diameter, and the ex vivo counts were 65 . It did contain metastasis after definitive pathology. The routine pathology indicated: (left) invasive ductal carcinoma of $1.8 \mathrm{~cm}$, grade II, ASLN (2/2), ALN (3/18), and IMSLN $(1 / 1)$. The final pathological stage for this male patient was pT1cN3bM0 IIIC.

\section{Discussion}

Because of the rarity of breast carcinoma in men, the feasibility of large prospective randomized trials for MBC is limited, and the standard treatment for male patients has been derived from the results of clinical trials performed in females. The major prognostic factors in $\mathrm{MBC}$ are the same as those found in women, with lymph node involvement being the most significant prognostic factor for both sexes. Among patients with known lymph node status, men were 1.6 times as likely to have lymph node involvement. ${ }^{5}$

Similarly to FBC, ASLNB also has proven accuracy and feasibility in $\mathrm{MBC} .{ }^{3}$ It could reliably predict the state of the axilla and avoid unnecessary axillary dissection which may lead to lymphedema, pain, and numbness of upper limb.

Accurate staging could not be achieved by depending on the status of the ALN alone both in MBC and FBC, as there are two main nodal regions of the breast (the ALN and the internal mammary lymph node [IMLN] $)^{6}$ and there is no significant difference of the pathways between males and females. ${ }^{7}$ IMLN metastasis has similar prognostic importance as that of $\mathrm{ALN},{ }^{8}$ and it was reported to be present in $18 \%$ to $33 \%$ (mean $23.4 \%$ ) of FBC patients, and metastasis 
exclusively situated in the IMLN occurs in $2 \%$ to $11 \%$ of patients. ${ }^{9}$ Patients with both axillary and internal mammary positive nodes have a very poor prognosis. ${ }^{10}$ If the IMSLN is positive, internal mammary radiotherapy should be performed, and staging achieved by the status of the ALN alone would lead to under-stage and under-/over-treatment. As we all know, extended radical mastectomy had been abandoned because it did not improve the survival of patients, and current imaging techniques, such as positron emission tomography/ computed tomography, ultrasound and magnetic resonance imaging, could only detect metastatic lesions larger than $5 \mathrm{~mm},{ }^{8}$ so we recommend IM-SLNB as a minimally invasive technique for the efficient evaluation of the status of IMSLN to obtain complete nodal staging. If there is a high clinical suspicion for IMLN involvement, biopsy needs to be performed to diagnose whether or not it is involved. Gennari et $\mathrm{al}^{11}$ first reported a patient with $\mathrm{MBC}$ who underwent both ASLNB and IM-SLNB in 2004. Although the IMSLN did not contain metastatic cancer, it helped to determine the stage of the breast cancer for this MBC patient.

IM-SLNB is safe and feasible, and it needs to be considered both in $\mathrm{MBC}$ and $\mathrm{FBC}$. Gnerlich et $\mathrm{al}^{12}$ reported no complications in their FBC study, and Caudle et a ${ }^{13}$ found only two intraoperative surgical complications, but both were resolved smoothly. Our current study on FBC indicates that the success rate of IM-SLNB is $85.8 \%$, and $9.4 \%$ had positive IMSLN ${ }^{14}$ In addition, only $5 \%$ of patients had intraoperative surgical complications, including injury to pleura and internal mammary artery. They were repaired intraoperatively, only one had a small pneumothorax on postoperative chest radiography that resolved without intervention and postoperative bleeding. The side effects are negligible when compared with the benefits.

IM-SLNB could guide adjuvant radiotherapy which is more important for MBC than FBC. The 2015 National Comprehensive Cancer Network Breast Cancer Clinical Practice Guidelines strongly recommend consideration of radiotherapy of IMLN for patients who are ALN positive after surgery. ${ }^{15}$ But many ALN positive patients did not have IMLN involvement. What is more, breast cancer occurs at an older age in males than in females, these patients may have more frequent cardiovascular and pulmonary comorbidities. ${ }^{16}$ Even though with the more modern radiotherapy techniques ${ }^{17}$ the toxicity to heart and lungs caused by radiotherapy is estimated to be less, special concern in $\mathrm{MBC}$ is still needed. Of note, in our study of women some patients with negative ALN did have IMLN involvement, and it might be the same in men. So, only relying on the status of ALN to perform internal mammary radiotherapy might lead to under-/over-treatment. IM-SLNB could make it possible to personalize treatment for patients thereby avoiding needless internal mammary radiation therapy. Cong et $\mathrm{al}^{8}$ suggested that patients with positive IMSLN should receive internal mammary radiotherapy, while it could be avoided in those with negative IMSLN. However, there is a need for further studies in IMSLN positive patients in order to confirm that addition of radiation to internal mammary site could improve outcomes.

The low visualization rate of IMSLN is the restriction for both clinical study and daily practice of IM-SLNB. ${ }^{8}$ A modified radiotracer injection technique (high volume, intraparenchymal, ultrasonographic guidance) was invented which broke through the bottle-neck of the low internal mammary visualization rate with traditional injection $(71 \%$ versus $13.8 \%, P<0.001)$ in FBC. ${ }^{18}$ IMSLN was only detected in two of the four MBC cases in this case report due to the lower visualization rate in males than in females, which may be associated with the fact that men have less glands than women. We recommend that $\mathrm{MBC}$ should be diagnosed with core needle biopsy rather than excisional biopsy to avoid gland damage.

\section{Conclusion}

We suggest that IM-SLNB should be performed in MBC patients with IMSLN detected by preoperative lymphoscintigraphy or intraoperative gamma-probe. IM-SLNB is safe and feasible, and it might help to determine nodal staging and the pathological status of IMSLN in MBC, in case of under-stage and under-/over-treatment. Further studies are needed to confirm the value of IM-SLNB both in female and male patients with breast cancer.

\section{Disclosure}

The authors have stated that they have no conflicts of interest in this work.

\section{References}

1. Piscuoglio S, Murray M, Ng CK, et al. The genomic landscape of male breast cancer. Presented at San Antonio Breast Cancer Symposium; December 12, 2014; San Antonio, TX.

2. Veronesi U, Paganelli G, Viale G, et al. A randomized comparison of sentinel node biopsy with routine axillary dissection in breast cancer. N Engl J Med. 2003;349(6):546-553.

3. Krag D, Julian TB, Harlow SP, et al. NSABP-32: Phase III, randomized trial comparing axillary resection with sentinal lymph node dissection: a description of the trial. Ann Surg Oncol. 2004;11 (3 Suppl):208S-210S.

4. Flynn LW, Park J, Patil SM, Cody HS 3rd, Port ER. Sentinel lymph node biopsy is successful and accurate in male breast carcinoma. J Am Coll Surg. 2008;206(4):616-621. 
5. Giordano SH, Cohen DS, Buzdar AU, Perkins G, Hortobagyi GN. Breast carcinoma in men: a population-based study. Cancer. 2004;101(1): $51-57$.

6. Turner-Warwick RT. The lymphatics of the breast. Br J Surg. 1959;46: 574-582.

7. Suami H, Pan WR, Mann GB, Taylor GI. The lymphatic anatomy of the breast and its implications for sentinel lymph node biopsy: a human cadaver study. Ann Surg Oncol. 2008;15(3):863-871.

8. Cong BB, Qiu PF, Wang YS. Internal mammary sentinel lymph node biopsy: minimally invasive staging and tailored internal mammary radiotherapy. Ann Surg Oncol. 2014;21(7):2119-2121.

9. van der Ent FW, Kengen RA, van der Pol HA, et al. Halsted revisited: internal mammary sentinel lymph node biopsy in breast cancer. Ann Surg. 2001;234(1):79-84.

10. Veronesi U, Cascinelli N, Bufalino R, et al. Risk of internal mammary lymph node metastases and its relevance on prognosis of breast cancer patients. Ann Surg. 1983;198(6):681-684.

11. Gennari R, Scelsi M, Greco B, Costa A. Complete nodal staging in male breast cancer. (Biopsy of axillary and internal mammary sentinel node). J Exp Clin Cancer Res. 2004;23(1):163-165.

12. Gnerlich JL, Barreto-Andrade JC, Czechura T, et al. Accurate staging with internal mammary chain sentinel node biopsy for breast cancer. Ann Surg Oncol. 2014;21(2):368-374.
13. Caudle AS, Yi M, Hoffman KE, et al. Impact of identification of internal mammary sentinel lymph node metastasis in breast cancer patients. Ann Surg Oncol. 2014;21(1):60-65.

14. Cong BB, Wang YS, Qiu PF, et al. A modified injection technique for internal mammary sentinel lymph node biopsy and its validation study. Presented at Asian Breast Cancer Conference; October 17, 2014; Taipei, Taiwan.

15. Gradishar WJ, Anderson BO, Balassanian R, et al. NCCN Clinical Practice Guidelines in Oncology breast carcinoma-V.2.2015. National Comprehensive Cancer Network. Available from: www.nccn.org/ patients. Accessed May 29, 2015.

16. Fentiman IS, Fourquet A, Hortobagyi GN. Male breast cancer. Lancet. 2006;367(9510):595-604.

17. Mast ME, van Kempen-Harteveld L, Heijenbrok MW, et al. Left-sided breast cancer radiotherapy with and without breath-hold: does IMRT reduce the cardiac dose even further? Radiother Oncol. 2013;108(2): 248-253.

18. Qiu PF, Liu JJ, Liu YB, et al. A modified technology could significantly improve the visualization rate of the internal mammary sentinel lymph nodes in breast cancer patients. Breast Cancer Res Treat. 2012;136(1): 319-321.
OncoTargets and Therapy

\section{Publish your work in this journal}

OncoTargets and Therapy is an international, peer-reviewed, open access journal focusing on the pathological basis of all cancers, potential targets for therapy and treatment protocols employed to improve the management of cancer patients. The journal also focuses on the impact of management programs and new therapeutic agents and protocols on

\section{Dovepress}

patient perspectives such as quality of life, adherence and satisfaction. The manuscript management system is completely online and includes a very quick and fair peer-review system, which is all easy to use. Visit http://www.dovepress.com/testimonials.php to read real quotes from published authors. 